\title{
TOWERS ON TREES
}

\author{
MARTIN GOLDSTERN, MARK J. JOHNSON, AND OTMAR SPINAS
}

(Communicated by Franklin D. Tall)

\begin{abstract}
ABSTRACr. We show that (under MA) for any c many dense sets in Laver forcing $\mathbb{L}$ there exists a $\sigma$-centered $Q \subseteq \mathbb{L}$ such that all the given dense sets are dense in $Q$. In particular, MA implies that $\mathbb{L}$ satisfies MA and does not collapse the continuum and the additivity of the Laver ideal is the continuum.

The same is true for Miller forcing and for Mathias forcing. In the case of Miller forcing this involves the correction of the wrong proof of Judah, Miller, and Shelah, Sacks, Laver forcing, and Martin's Axiom, Arch. Math. Logic 31 (1992), Theorem 4.1, p. 157.
\end{abstract}

\section{INTRODUCTION}

In [V] a model for ZFC has been constructed where Martin's Axiom holds for perfect set forcing ( $\mathbb{S}$ ) and the continuum is arbitrarily large. It is clear that for this we cannot just iterate forcing with $\mathbb{S}$ since then we would have to use countable supports and hence would never get the continuum larger than $\omega_{2}$. Velickovic's method is roughly the following. For $P$ a forcing notion and $\mathscr{C}$ a class (or property) of forcing notions consider the following statement $\mathscr{C}(P)$ :

Whenever $\mathscr{D}$ is a family of at most $2^{\aleph_{0}}$ many dense sets in $P, p \in P$, then there is a $Q \in \mathscr{C}, p \in Q \subseteq P$, such that for every $D \in \mathscr{D}, D \cap Q$ is dense in $Q$.

Velickovic constructs a finite support iteration where each iterand is alternately either a carefully chosen finite support product of ccc suborderings of $\mathbb{S}$ or an arbitrary ccc forcing. The delicate point is to show that each of those finite support products is ccc. In that way a model is obtained where simultaneously $\operatorname{ccc}(\mathbb{S})$ (where $\operatorname{ccc}$ is the class of all partial orderings satisfying the countable chain condition) and MA hold. Then clearly $M A(\mathbb{S})$ is also true.

Velickovic proves that MA is not enough to get $M A(\mathbb{S})$, a fortiori MA does not imply $\operatorname{ccc}(\mathbb{S})$. In $[V]$ it is even shown that PFA implies the failure of $\operatorname{ccc}(\mathbb{S})$.

Velickovic indicates that a construction similar to that he does for $\mathbb{S}$ works for all tree-like forcings. Here we show that at least for Mathias, Laver, and

Received by the editors September 1, 1992 and, in revised form, January 4, 1993.

1991 Mathematics Subject Classification. Primary 03E05; Secondary 03E50, 06A07.

Key words and phrases. Laver forcing, Miller forcing, Martin's axiom, capturing density.

The first and third authors are grateful to the Basic Research Foundation of the Israel Academy of Science. 
Miller forcing there is no need for such a construction. Writing $\mathfrak{t}$ for the length of the shortest maximal tower in $[\omega]^{\omega} /$ finite, we show:

Theorem. (a) $\mathfrak{t}=\mathfrak{c}$ implies $\sigma$-centered(Mathias).

(b) $\mathfrak{t}=\mathbf{c}$ implies $\sigma$-centered(Laver).

(c) $M A(\sigma$-centered $)$ implies $\sigma$-centered(Miller).

Our method of proof uses ideas from [JMS]. As corollaries of the theorem we obtain that if MA holds, then first, Mathias, Laver, and Miller forcings do not collapse the continuum-in the case of Laver forcing our proof is a slight improvement of the result "MA( $\sigma$-centered) implies that Laver forcing does not collapse cardinals" from [JMS] (recall that MA( $\sigma$-centered) easily implies $t=c$ ), for Miller forcing this provides a correction to the proof from [JMS]. Second, MA holds for these forcings, and third, the ideals associated with them have additivity continuum.

\section{CAPTURING Density}

2.1. Definition. If $P$ is a forcing notion, $D \subseteq P$ dense, $Q \subseteq P$, then we say that $Q$ captures the density of $D$ if $D \cap Q$ is dense in $Q$.

If $\mathscr{D}$ is a family of dense sets, then we say that $Q$ captures the density of $\mathscr{D}$ if and only if $Q$ captures the density of each $D \in \mathscr{D}$.

2.2. Definition. If $\mathscr{C}$ is a class of forcing notions, $P$ a forcing notion, then we write $\mathscr{C}(P)$ for the statement

Whenever $\mathscr{D}$ is a family of at most $2^{\aleph_{0}}$ many dense sets in $P, p \in P$, then there is a $Q \in \mathscr{C}, p \in Q \subseteq P$, which captures the density of $\mathscr{D}$.

(This notion is due to [V].)

The motivation for this concept is given by the following two facts:

2.3. Fact. If $\operatorname{MA}(\mathscr{C})$ and $\mathscr{C}(P)$, then $\operatorname{MA}(P)$.

(Here, $\mathrm{MA}(P)$ means that for any collection of less than $2^{\aleph_{0}}$ many dense sets in $P$ there exists a filter intersecting all of them. $M A(\mathscr{C})$ means that $\operatorname{MA}(P)$ holds for all $P$ in $\mathscr{C}$. Thus, the usual MA is MA(ccc).)

2.4. Fact. If $\operatorname{ccc}(P)$, then $P$ does not change the cofinality of any cardinal $\leq 2^{\aleph_{0}}$.

Proof. Assume $\operatorname{ccc}(P)$; let $\lambda<\operatorname{cf}(\kappa), \kappa \leq \mathfrak{c}$; and assume that

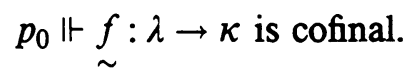

For $\alpha<\lambda$ let

$$
D_{\alpha}:=\left\{p: p \perp p_{0} \text { or } \exists \beta p \Vdash \underset{\sim}{f}(\check{\alpha})=\check{\beta}\right\},
$$

and for $\gamma<\kappa$ let

$$
E_{\gamma}:=\left\{p: p \perp p_{0} \text { or } \exists \alpha \exists \beta>\gamma p \Vdash \underset{\sim}{f}(\check{\alpha})=\beta\right\} .
$$

Clearly all sets $D_{\alpha}$ and all sets $E_{\gamma}$ are dense. 
Let $Q \subseteq P$ be a ccc set capturing the density of all $D_{\alpha}$ and all $E_{\gamma}$, with $p_{0} \in Q$. Let $G \subseteq Q$ be generic, $p_{0} \in G$. Since $Q$ is ccc,

$$
V[G] \vDash \operatorname{cf}(\kappa)>\lambda .
$$

Working in $V[G]$, let

$$
R:=\left\{(\alpha, \beta): \alpha<\lambda, \beta<\kappa, \exists p \in G, V \vDash p \Vdash_{P} \underset{\sim}{f}(\check{\alpha})=\check{\beta}\right\} .
$$

It is easy to see that (in $V[G]$ ):

(1) $R$ is a function (with domain $\lambda$ ) and

(2) the range of $R$ is cofinal in $\kappa$.

So $V[G] \vDash \operatorname{cof}(\kappa) \leq \lambda$, a contradiction.

\section{LAVER FORCING}

3.1. Theorem. $\mathfrak{t}=\mathfrak{c}$ implies $\sigma$-centered(Laver).

3.2. Notation. $\mathbb{L}$ will be the set of conditions in Laver forcing. For $p \in \mathbb{L}$ we let $\operatorname{stem}(p) \in{ }^{<\omega} \omega$ be the stem of $p$ and $p^{-}:=\{s \in p: \operatorname{stem}(p) \subseteq s\}$. $\operatorname{succ}_{p}(s):=\{i: s \sim i \in p\}$.

Note that we write forcing "upwards": $p \geq q$ if and only if $p \subseteq q$ if and only if $p$ "extends" $q$ if and only if $p$ "has more information than" $q$.

We write $p \geq^{0} q$ if and only if $p \geq q$ and $\operatorname{stem}(p)=\operatorname{stem}(q)$.

3.3. Definition. Let $\kappa$ be an ordinal. A sequence $\underline{A}=\left(A_{\alpha}: \alpha<\kappa\right)$ is called a tower of height $\kappa$ if

(1) $\forall \alpha: A_{\alpha} \subseteq \omega$ and

(2) $\forall \alpha<\beta: A_{\beta} \subseteq^{*} A_{\alpha}$, i.e., $A_{\beta}-A_{\alpha}$ is finite.

$\underline{A}$ is called maximal if there is no set $A_{\kappa}$ such that $\left(A_{\alpha}: \alpha<\kappa+1\right)$ is a tower.

We let $\mathfrak{t}$ be the minimal height of a maximal tower. It is well kncwn that MA (or even MA( $\sigma$-centered)) implies $\mathfrak{t}=\mathfrak{c}$.

3.4. Definition. Let $\bar{A}=\left(A_{s}: s \in{ }^{<\omega} \omega\right)$ be a family of infinite sets. We let

$$
\mathbb{L}_{\bar{A}}:=\left\{p \in \mathbb{L}:\left(\forall s \in p^{-}\right) A_{s} \subseteq^{*} \operatorname{succ}_{p}(s)\right\} .
$$

3.5. Definition. For $\bar{A}$ and $\bar{B}$ as in 3.4 we write $\bar{A} \geq^{*} \bar{B}$ if $\forall s \in{ }^{<\omega} \omega A_{s} \subseteq^{*}$ $B_{s}$.

Clearly, if $p, q \in \mathbb{L}_{\bar{A}}$ and $\operatorname{stem}(p)=\operatorname{stem}(q)$, then also $p \cap q \in \mathbb{L}_{\bar{A}}$. Hence $\mathbb{L}_{\bar{A}}$ is $\sigma$-centered.

Proof of Theorem 3.1. Let $\mathscr{D}$ be a collection of $\mathfrak{c}$ many dense sets in $\mathbb{L}$, and $p_{0}$ a condition, and enumerate $\mathbb{L} \times \mathscr{D}$ as

$$
\mathbb{L} \times \mathscr{D}=\left\{\left(p_{\alpha}, D_{\alpha}\right): \alpha<\mathfrak{c}\right\} .
$$

We will define a sequence

$$
\left(\bar{A}^{\alpha}: \alpha<\mathfrak{c}\right) \quad \bar{A}^{\alpha}=\left(A_{s}^{\alpha}: s \in \epsilon^{<\omega} \omega\right)
$$

satisfying 
The construction proceeds by induction on $\alpha$. For $\alpha=0$, let

$$
A_{s}^{\alpha}:= \begin{cases}\omega & \text { if } s \notin\left(p_{0}\right)^{-} \\ \operatorname{succ}_{p_{0}}(s) & \text { if } s \in\left(p_{0}\right)^{-}\end{cases}
$$

If $\alpha$ is a limit, we define $A_{s}^{\alpha}$ such that $(*)$ is satisfied, using $\mathfrak{t}=\mathfrak{c}$.

If $\alpha=\beta+1$, we distinguish two cases:

Case 1. For no $\bar{A} \geq^{*} \bar{A}^{\beta}, p_{\beta} \in \mathbb{L}_{\bar{A}}$. In this case we let $A_{s}^{\alpha}=A_{s}^{\beta}$ for all $s$.

Case 2. Otherwise, let $s_{\beta}:=\operatorname{stem}\left(p_{\beta}\right)$ and

$$
p_{\beta}^{\prime}:=\left\{s \in p_{\beta}: \forall i \in \operatorname{dom}(s)\left(i<\left|s_{\beta}\right| \text { or } s(i) \in A_{s i i}^{\beta}\right)\right\} .
$$

We claim that $p_{\beta}^{\prime}$ is a condition, $p_{\beta}^{\prime} \geq p_{\beta}$, and $\operatorname{stem}\left(p_{\beta}^{\prime}\right)=\operatorname{stem}\left(p_{\beta}\right)$, and for all $s \in p_{\beta}^{\prime}$ we have $\operatorname{succ}_{p_{\beta}^{\prime}}(s)=\operatorname{succ}_{p_{\beta}}(s) \cap A_{s}^{\beta}$. For this it is enough to see that for each $s \in p_{\beta}^{-}, \operatorname{succ}_{p_{\beta}}(s) \cap A_{s}^{\beta}$ is infinite. If not, then there is $s \in p_{\beta}^{-}$such that

$$
\operatorname{succ}_{p_{\beta}}(s) \cap A_{s}^{\beta} \text { is finite. }
$$

Since $p_{\beta} \in \mathbb{L}_{\bar{A}}$ for some $\bar{A} \geq^{*} \bar{A}^{\beta}$ and hence $A_{s} \subseteq^{*} \operatorname{succ}_{p_{\beta}}(s)$, we conclude that $A_{s} \cap A_{s}^{\beta}$ is finite-a contradiction, since $A_{s} \subseteq^{*} A_{s}^{\beta}$. Now we can find a condition $q_{\beta} \geq p_{\beta}^{\prime}, q_{\beta} \in D_{\beta}$ and define

$$
A_{s}^{\alpha}:= \begin{cases}\operatorname{succ}_{q_{\beta}}(s) & \text { if } s \in q_{\beta}^{-}, \\ A_{s}^{\beta} & \text { otherwise. }\end{cases}
$$

Note that $q_{\beta} \in \mathbb{L}_{\bar{A}^{\alpha}}$.

This concludes the construction of the sequence of $\bar{A}^{\alpha}$ 's.

Now we let $Q:=\bigcup_{\alpha<c} \mathbb{L}_{\bar{A}^{\alpha}}$, and we claim that:

(1) $\forall \beta<\alpha: \mathbb{L}_{\bar{A}^{\beta}} \subseteq \mathbb{L}_{\bar{A}^{\alpha}}$,

(2) $Q$ is $\sigma$-centered, and

(3) $Q$ captures the density of $\mathscr{D}$.

The proof of these claims will finish the proof of the theorem, since clearly $p_{0} \in \mathbb{L}_{\tilde{A}^{0}} \subseteq Q$.

Proof of (1). Obvious.

Proof of (2). If $p, q \in Q$ with $\operatorname{stem}(p)=\operatorname{stem}(q)$, then there is $\alpha$ such that $p, q \in \mathbb{L}_{\bar{A}^{\alpha}}$. So $p \cap q \in \mathbb{L}_{\bar{A}^{\alpha}} \subseteq Q$.

Proof of (3). Let $D \in \mathscr{D}, p \in Q$. We claim that there is $q \in Q \cap D, q \geq p$.

Assume that $(D, p)=\left(D_{\beta}, p_{\beta}\right)$. We only have to show that $q_{\beta}$ is well defined. But this is obvious since by $p_{\beta} \in Q$ at stage $\beta+1$ we must have been in Case 2.

3.6. Remark. A similar construction, using only one tower instead of a system of towers, shows the analogous result for Mathias forcing.

3.7. Definition. Let $\ell^{0}$ denote the $\sigma$-ideal of all $X \subseteq \omega^{\omega}$ such that $\forall p \in$ $\mathbb{L} \exists q \in \mathbb{L}(q \geq p$ and $[q] \cap X=\varnothing)$. Remember that $\operatorname{add}\left(\ell^{0}\right)$ is the minimal cardinality of a family of members of $\ell^{0}$ whose union does not belong to $\ell^{0}$. 
3.8. Corollary. $M A$ implies add $\left(\ell^{0}\right)=\mathfrak{c}$.

Proof. Let $\left\langle X_{\alpha}: \alpha<\kappa\right\rangle$ be a family in $\ell^{0}$ and $\kappa<\mathfrak{c}$. Let $D_{\alpha}=\{p \in \mathbb{L}$ : $\left.[p] \cap X_{\alpha}=\varnothing\right\}$. Observe that $D_{\alpha}$ is $\geq^{0}$-dense in $\mathbb{L}$, i.e., $\forall p \in \mathbb{L} \exists q \in D_{\alpha}(q \geq p$ and $\operatorname{stem}(p)=\operatorname{stem}(q))$. Now choose a ccc $Q \subseteq \mathbb{L}$ which captures the density of $\left\langle D_{\alpha}: \alpha<\kappa\right\rangle$.

Define "amoeba forcing" for $Q$ as follows: $\mathscr{A}(Q)=\{(p, n): p \in Q, 1 \leq$ $n<\omega\}$ ordered by

$(p, n) \geq(q, m)$ if and only if $p \geq q$ and $n \geq m$ and $\forall i<m p(i)=q(i)$

where $p(\cdot)$ is the canonical enumeration of $p^{-}$. In particular, this implies $\operatorname{stem}(p)=p(0)=q(0)=\operatorname{stem}(q)$.

If we know that $D_{\alpha}^{*}=\left\{(p, n): p \in D_{\alpha}\right\}$ is dense in $\mathscr{A}(Q)$ and $\mathscr{A}(Q)$ is ccc, then applying MA to $\mathscr{A}(Q)$ and $\left\langle D_{\alpha}^{*}: \alpha<\kappa\right\rangle$ we could obtain a Laver tree whose branches are disjoint from every $X_{\alpha}$, and since the whole argument could be done above a given tree, we would be done.

But, in fact, without loss of generality we may assume that each $D_{\alpha}^{*}$ is dense. For in the construction of $Q$ in the proof of 3.1,using the observation that each $D_{\alpha}$ is $\geq^{0}$-dense, at stage $\alpha=\beta+1$ we may choose $q_{\beta} \in D_{\beta}$ such that $q_{\beta} \geq^{0} p_{\beta}$. But then each $D_{\alpha}$ is $\geq^{0}$-dense in $Q$ and hence, as can be easily checked, $D_{\alpha}^{*}$ is dense in $\mathscr{A}(Q)$. Furthermore, two conditions in $\mathscr{A}(Q)$, say $(p, n),(q, m)$ are compatible provided $\operatorname{stem}(p)=\operatorname{stem}(q), n=m$, and $\forall i<n p(i)=q(i)$. This is true since two conditions in $Q$ with the same stem $s$ have an extension in $Q$ with stem $s$. This shows that $\mathscr{A}(Q)$ is even $\sigma$-centered.

\section{MILLER FORCING}

4.1. Theorem. $M A(\sigma$-centered) implies $\sigma$-centered(Miller).

4.2. Notation. $\mathbb{F}$ is Miller forcing (also called rational perfect sets). Conditions $p \in \mathbb{F}$ are superperfect trees $p \subseteq{ }^{<\omega} \omega$, that is, trees which have infinite splitting along every branch. We will consider only the dense subset of superperfect trees $p$ with the property

$$
\forall s \in p:\left|\operatorname{succ}_{p}(s)\right| \in\left\{1, \aleph_{0}\right\} .
$$

For $p \in \mathbb{F}$, $\operatorname{split}(p)$ is the set of splitting nodes of $p$, with smallest element $\operatorname{stem}(p)$. This set is also partially ordered by $\subseteq$, and we write $\operatorname{Succ}_{p}(s)$ for the set of direct successors of $s$ in $\operatorname{split}(p)$.

Again we recall that $p \geq q$ means " $p$ is stronger than $q$ ", i.e., $p \subseteq q$.

In 4.3-4.10 we modify the argument of [JMS] to find a $\leq^{*}$ which is transitive.

4.3. Definition. Call a sequence $\left\langle P_{s}: s \in{ }^{<\omega} \omega\right\rangle$ good if and only if

(1) each $P_{s} \subseteq{ }^{<\omega} \omega$ is infinite,

(2) $t \in P_{s}$ implies $s \subset t$, and

(3) for $s \in \omega^{n}$ if $t, t^{\prime} \in P_{s}$ and $t \neq t^{\prime}$, then $t(n) \neq t^{\prime}(n)$.

4.4. Definition. Given any good sequence $\bar{P}=\left\langle P_{s}: s \in{ }^{<\omega} \omega\right\rangle$ we determine $\left\langle p_{s} \in \mathbb{F}: s \in{ }^{<\omega} \omega\right\rangle$ as follows. For each $s$ let $S$ be the smallest subset of ${ }^{<\omega} \omega$ such that $s \in S$ and if $t \in S$ then $P_{t} \subseteq S$. Then $p_{s}$ is the unique condition in $\mathbb{F}$ such that $S=\operatorname{split}\left(p_{s}\right)$. In other words, $s=\operatorname{stem}\left(p_{s}\right)$, and if $t \in \operatorname{split}\left(p_{s}\right)$ then $\operatorname{Succ}_{p_{s}}(t)=P_{t}$.

If $\bar{Q}, P^{\prime}$, etc., are good, then $q_{s}, p_{s}^{\prime}$, etc., will be defined similarly. 
4.5. Definition. Define $\left\langle P_{s}: s \in{ }^{<\omega} \omega\right\rangle \geq\left\langle Q_{s}: s \in{ }^{<\omega} \omega\right\rangle$ if and only if $p_{s} \subseteq q_{s}$ for each $s \in{ }^{<\omega} \omega$. An equivalent definition would be: For all $s, P_{s} \subseteq \operatorname{split}\left(q_{s}\right)$.

4.6. Definition. Define $\left\langle P_{s}: s \in \epsilon^{<\omega} \omega\right\rangle \approx\left\langle Q_{s}: s \in{ }^{<\omega} \omega\right\rangle$ if and only if

(a) $\forall s P_{s}={ }^{*} Q_{s}$ and

(b) $\forall{ }^{\infty} s P_{s}=Q_{s}$.

Clearly this is an equivalence relation.

4.7. Definition. Let $\bar{P}$ and $\bar{Q}$ be good. We will write $\bar{P} \geq \geq^{*} \bar{Q}$ if and only if

(1) There exists $\bar{P}^{\prime} \approx \bar{P}$ such that $\bar{P}^{\prime} \geq \bar{Q}$.

4.8. Lemma. If there exists $\bar{Q}^{\prime}$ such that $\bar{P} \geq \bar{Q}^{\prime} \approx \bar{Q}$; then $\bar{P} \geq \geq^{*} \bar{Q}$, i.e., there is $\bar{P}^{\prime}$ such that $\bar{P} \approx \bar{P}^{\prime} \geq Q$. Moreover, we can choose $\bar{P}^{\prime}$ such that $\bar{P}^{\prime} \geq \bar{P}$.

Schematically, we can write this as follows:

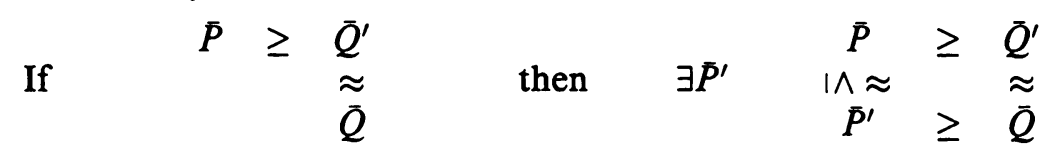

Proof. Assume we have $\bar{P} \geq \bar{Q}^{\prime} \approx \bar{Q}$. Recall that for all $s \in{ }^{<\omega} \omega$ we have $P_{s} \subseteq \operatorname{split}\left(q_{s}\right)$. We can define $\bar{P}^{\prime}$ by

$$
P_{s}^{\prime}:=P_{s} \cap \operatorname{split}\left(q_{s}\right) \text {. }
$$

Fix $s \in{ }^{<\omega} \omega$. To understand why $P_{s}={ }^{*} P_{s}^{\prime}$ we consider the function $\rho_{s}$ defined on $\operatorname{split}\left(q_{s}^{\prime}\right)-\operatorname{split}\left(q_{s}\right)$ as follows:

For any $t \in \operatorname{split}\left(q_{s}^{\prime}\right)$ we can find a finite sequence $s=r_{0} \subseteq r_{1} \subseteq \cdots \subseteq r_{n}=t$, where for all $k<n$ we have $r_{k+1} \in Q_{r_{k}}^{\prime}$.

For $t \in \operatorname{split}\left(q_{s}^{\prime}\right)-\operatorname{split}\left(q_{s}\right)$ we let

$$
\rho_{s}(t):=\text { the minimal } r_{k+1} \text { with } r_{k+1} \notin Q_{r_{k}}
$$

Note that $s \subset \rho_{s}(t) \subseteq t$, so $\rho_{s}(t)(|s|)=t(|s|)$, hence (by 4.3(3)) the function $\rho_{s}\left\lceil P_{s}\right.$ is one-to-one. Hence

$$
\left|\left\{t \in P_{s}: t \notin \operatorname{split}\left(q_{s}\right)\right\}\right| \leq \sum_{r}\left|Q_{r}^{\prime}-Q_{r}\right|=\text { finite. }
$$

So for all $s$ we have $P_{s}={ }^{*} P_{s}^{\prime}$, in particular, we get that $\bar{P}^{\prime}$ is good.

Let $A:=\left\{t \in{ }^{<\omega} \omega: \exists s \in{ }^{<\omega} \omega: t \subseteq s \wedge Q_{s} \neq Q_{s}^{\prime}\right\} . A$ is finite (and downward closed), and for $s \notin A$ we have $q_{s}=q_{s}^{\prime}$ and hence $P_{s}^{\prime}=P_{s}$. So $\bar{P}^{\prime} \approx \bar{P}$. Finally, it is clear that $\bar{P}^{\prime} \geq \bar{Q}$ and $\bar{P}^{\prime} \geq \bar{P}$.

4.9. Remark. This shows that

$$
P \geq^{*} \bar{Q} \text { if and only if there is } \bar{P}^{\prime} \approx \bar{P}, \bar{P}^{\prime} \geq \bar{Q}, P^{\prime} \geq \bar{P} \text {. }
$$

Proof. If $\bar{P} \approx P_{1} \geq \bar{Q}$, then we can apply 4.8 to the relation $\bar{P} \geq \bar{P} \approx \bar{P}_{1}$ and get $P^{\prime}$ such that

$$
\begin{aligned}
\bar{P} & \geq \bar{P} \\
\wedge \approx & \approx \\
\bar{P}^{\prime} & \geq \bar{P}_{1} \geq \bar{Q}
\end{aligned}
$$

4.10. Corollary. (a) $\leq^{*}$ is transitive.

(b) If $P_{1} \geq^{*} P_{2} \geq^{*} \cdots \geq^{*} P_{n}$, then there exists $\bar{P}^{*}$ such that for $i=1, \ldots, n$ we have $P^{*} \geq P_{i}$. 
Proof. If $\bar{P} \geq^{*} \bar{Q} \geq^{*} \bar{R}$, then there are $\bar{P}^{\prime}$ and $\bar{Q}^{\prime}$ such that

$$
\bar{P} \approx \bar{P}^{\prime} \geq \bar{Q} \approx \bar{Q}^{\prime} \geq \bar{R} .
$$

By 4.9 we may assume $\bar{P} \leq \bar{P}^{\prime}$. By 4.8 we can find $\bar{P}^{*}$ such that

$$
\begin{aligned}
& \bar{P} \\
& 1 \wedge \approx \\
& { }_{\mid \wedge \approx} \bar{P}^{\prime} \geq \bar{Q} \\
& \bar{P}^{*} \geq \bar{Q}^{\prime} \geq \bar{R}
\end{aligned}
$$

This proves (a), and also (b) for the case $n=3$. For general $n$ the proof of (b) is similar.

4.11. Fact. For $\bar{P}$ good, $r \in \mathbb{F}$, the following are equivalent:

(1) $\forall s \in \operatorname{split}(r): \operatorname{split}\left(p_{s}\right) \subseteq \operatorname{split}(r)$.

(2) $\forall s \in \operatorname{split}(r): P_{s} \subseteq \operatorname{split}(r)$.

(3) $\forall s \in \operatorname{split}(r): \operatorname{split}\left(p_{s}\right) \subseteq r$.

(4) $\forall s \in \operatorname{split}(r): p_{s} \subseteq r$.

Proof. (1) $\Rightarrow(2)$ As $P_{s} \subseteq \operatorname{split}\left(p_{s}\right)$ for all $s$.

(2) $\Rightarrow(3)$ By induction on the height of $t \in \operatorname{split}\left(p_{s}\right)$ we can prove $t \in$ $\operatorname{split}(r)$.

(3) $\Rightarrow(4)$ As every node in $p_{s}$ is below some node in $\operatorname{split}\left(p_{s}\right)$ and hence also in $r$.

$(4) \Rightarrow(1)$ As splitting points of a stronger condition are also splitting points of the weaker condition.

4.12. Definition. For $\bar{P}$ good, we let

$$
\mathbb{F}_{P}:=\left\{r: \exists \bar{P}^{\prime} \approx \bar{P}, \forall s \in \operatorname{split}(r): \operatorname{split}\left(p_{s}^{\prime}\right) \subseteq \operatorname{split}(r)\right\}
$$

4.13. Fact. (a) $\mathbb{F}_{P}$ is $\sigma$-centered.

(b) If $\bar{P} \approx \bar{Q}$, then $\mathbb{F}_{P}=\mathbb{F}_{\bar{Q}}$.

(c) If $\bar{P} \geq * \bar{Q}$, then $\mathbb{F}_{P} \supseteq \mathbb{F}_{Q}$.

Proof. For (a), assume $r^{1}, r^{2} \in \mathbb{F}_{P}$, witnessed by $P^{1}, P^{2}$ respectively, and $\operatorname{stem}\left(r^{1}\right)=\operatorname{stem}\left(r^{2}\right)=s$. Since $\bar{P}^{1} \approx \bar{P}^{2}$, we may find $P_{s}^{3}$ such that $P_{s}^{3}={ }^{*} P_{s}^{i}$ for $i=1,2$ and $\forall t \in P_{s}^{3} \forall u\left(t \subseteq u \rightarrow P_{u}^{1}=P_{u}^{2}\right)$. Define $P_{t}^{3}=P_{t}^{1}$ for $t \neq s$. Now clearly we have $p_{s}^{3} \in \mathbb{F}_{P}$, and $p_{s}^{3}$ extends $r^{1}, r^{2}$. (b) can easily be checked. For (c), consider $r \in \mathbb{F}_{Q}$. By hypothesis find $\bar{P}^{\prime}, \bar{Q}^{\prime}$ such that $P \approx$ $\bar{P}^{\prime} \geq \bar{Q} \approx \bar{Q}^{\prime}$, and $\forall s \in \operatorname{split}(r)$ we have $q_{s}^{\prime} \subseteq r$. By 4.8 we can find $P^{\prime \prime} \approx \bar{P}^{\prime}$, $\bar{Q}^{\prime} \leq \bar{P}^{\prime \prime}$. So $\forall s \in \operatorname{split}(r)$ we have $p_{s}^{\prime \prime} \subseteq q_{s}^{\prime} \subseteq r$, hence $r \in \mathbb{F}_{P^{\prime \prime}}=\mathbb{F}_{P}$.

4.14. Lemma. For all $p \in \mathbb{F}$, all good $\bar{P}$, and all dense sets $D \subseteq \mathbb{F}$ : If there is $\bar{Q} \geq^{*} \bar{P}$ such that $p \in \mathbb{F}_{Q}$, then there is $\bar{Q} \geq^{*} \bar{P}, p \in \mathbb{F}_{Q}$, such that for some $q \geq p$ we have $q \in D \cap \mathbb{F}_{Q}$. Moreover, if $D$ is $\geq^{0}$-dense, then we may assume $\operatorname{stem}(p)=\operatorname{stem}(q)$.

Proof. Let $\bar{Q}^{0} \geq^{*} \bar{P}$ such that $p \in \mathbb{F}_{Q^{0}}$. Find $\bar{Q}^{1} \approx \bar{Q}^{0}$ such that $\forall s \in \operatorname{split}(p)$, $\operatorname{split}\left(q_{s}^{1}\right) \subseteq \operatorname{split}(p)$. (So also $\bar{Q}^{1} \geq^{*} \bar{P}$.) Find $q \in D, q \subseteq q_{\text {stem }(p)}^{1}$. So $\operatorname{split}(q) \subseteq \operatorname{split}(p)$. 
Now define $\bar{Q}$ as follows:

(1) If $s \in \operatorname{split}(q)$, then $Q_{s}:=\operatorname{Succ}_{q}(s)$.

(2) Otherwise, $Q_{s}:=Q_{s}^{1}$.

So in any case we have $Q_{s} \subseteq \operatorname{split}\left(q_{s}^{1}\right)$ and hence $\bar{Q} \geq \bar{Q}^{1} \geq^{*} \bar{P}$, and clearly $q \in \mathbb{F}_{Q}$.

4.15. Lemma. Assume $M A_{\kappa}(\sigma$-centered $)$. If $\left(\bar{P}_{\alpha}: \alpha<\kappa\right)$ is $a \geq^{*}$-descending sequence, then there exists $\bar{P}_{\kappa}$ such that for all $\alpha, \bar{P}_{\kappa} \geq^{*} \bar{P}_{\alpha}$.

Proof. Given a sequence $\left(\bar{P}^{\alpha}: \alpha<\kappa\right)$, we define the following forcing notion: Elements are of the form $\left(F, T_{s}: s \in S\right)$, where $F$ is a finite subset of $\kappa, S$ a finite subset of ${ }^{<\omega} \omega$, and each $T_{s}$ a finite subset of ${ }^{<\omega} \omega$ with

(i) $t \in T_{s}$ implies $s \subset t$ and

(ii) for $s \in \omega^{n}$, if $t, t^{\prime} \in T_{s}$ and $t \neq t^{\prime}$, then $t(n) \neq t^{\prime}(n)$.

We let $\left(F, T_{s}: s \in S\right) \leq\left(F^{\prime}, T_{s}^{\prime}: s \in S^{\prime}\right)$ if and only if

(a) $F \subseteq F^{\prime}$,

(b) $S \subseteq S^{\prime}$,

(c) $\forall s \in S: T_{s} \subseteq T_{s}^{\prime}$, and

(d) $\forall s \in S^{\prime} \forall \alpha \in F: T_{s}^{\prime}-T_{s} \subseteq \operatorname{split}\left(p_{s}^{\alpha}\right)$ where we let $T_{s}=\varnothing$ for $s \notin S$.

This forcing is $\sigma$-centered (since conditions with the same $\left(T_{s}: s \in S\right)$ are always compatible), and a generic filter $G$ describes a good $\bar{P}$ via

$$
P_{t}:=\left\{\eta \in{ }^{<\omega} \omega: \exists\left(F, T_{s}: s \in S\right) \in G, t \in S, \eta \in T_{t}\right\} .
$$

To check that each $P_{t}$ is infinite, we use 4.10 (b) and a density argument.

Similarly as in the proof for Laver forcing, 4.15 and 4.14 imply that MA (or indeed $\mathrm{MA}(\sigma$-centered $))$ implies $\operatorname{ccc}(\mathbb{F})$ and that the additivity of the Miller ideal is $c$.

\section{REFERENCES}

[JMS] H. Judah, A. Miller, and S. Shelah, Sacks, Laver forcing, and Martin's Axiom, Arch. Math. Logic 31 (1992).

[L] R. Laver, On the consistency of Borel's conjecture, Acta Math. 137 (1976), 151-169.

[Ma] A. R. D. Mathias, Happy families, Ann. of Math. Logic 12 (1977), 59-111.

[Mi] A. Miller, Rational perfect set forcing, Contemp. Math., vol. 31, Amer. Math. Soc., Providence, RI, 1984, pp. 143-159.

[V] B. Velickovic, ccc posets of perfect trees, Compositio Math. 79 (1991), 279-294.

Department of Mathematics, Bar Ilan University, 52900 Ramat Gan, IsRael Current address: Technische Universität, Wiedner Hauptstr 8/118, A-1040 Wien, Austria E-mail address: goldstrn@email.turien.ac.at

Department of Mathematics, University of Wisconsin, Madison, Wisconsin 53706 E-mail address: mjohnsonemath. visc.edu

Department of Mathematics, Hebrew University of Jerusalem, Givat Ram, Israel Current address: Abteilung Mathematik, ETH-Zentrum, 8092 Zürich, Switzerland E-mail address: spinas@math.ethz.ch 